\title{
HYPERSURFACES OF LORENTZIAN PARA-SASAKIAN MANIFOLDS
}

\author{
SELCEN YÜKSEL PERKTAŞ, EROL KILIÇ and SADIK KELEŞ
}

\begin{abstract}
In this paper we study the invariant and noninvariant hypersurfaces of $(1,1,1)$ almost contact manifolds, Lorentzian almost paracontact manifolds and Lorentzian para-Sasakian manifolds, respectively. We show that a noninvariant hypersurface of an $(1,1,1)$ almost contact manifold admits an almost product structure. We investigate hypersurfaces of affinely cosymplectic and normal $(1,1,1)$ almost contact manifolds. It is proved that a noninvariant hypersurface of a Lorentzian almost paracontact manifold is an almost product metric manifold. Some necessary and sufficient conditions have been given for a noninvariant hypersurface of a Lorentzian para-Sasakian manifold to be locally product manifold. We establish a Lorentzian para-Sasakian structure for an invariant hypersurface of a Lorentzian para-Sasakian manifold. Finally we give some examples for invariant and noninvariant hypersurfaces of a Lorentzian para-Sasakian manifold.
\end{abstract}

\section{Introduction}

Hypersurfaces of an almost contact manifold have been studied by D. E. Blair [2], S. S. Eum [5], S. I. Goldberg and K. Yano [7], G. D. Ludden [8] and others. In 1970, S. I. Goldberg and K. Yano [7] defined noninvariant hypersurfaces of almost contact manifolds. A hypersurface such that the transform of a tangent vector of the hypersurface by the tensor $\varphi$ defining the almost contact structure is never tangent to the hypersurface is called a noninvariant hypersurface of the almost contact manifold [7]. The authors [7] showed that a noninvariant hypersurface of an almost contact manifold admits an almost complex structure and a distinguished 1-form induced by the contact form of the manifold. They also investigated noninvariant hypersurfaces of an almost contact metric manifold.

In 1976, I. Sato [13] studied a structure similar to the almost contact structure, namely almost paracontact structure. In [1], T. Adati studied hypersurfaces of an almost paracontact manifold. A. Bucki [3] considered hypersurfaces of an almost $r$-paracontact Riemannian manifold. Some properties of invariant hypersurfaces of an almost $r$-paracontact Riemannian manifold were investigated in [4] by A. Bucki and A. Miernowski. Moreover, in [10], I. Mihai and K. Matsumoto studied submanifolds of an almost $r$-paracontact Riemannian

Received 13 January 2010, in final form 23 August 2010. 
manifold of P-Sasakian type. In [6] the authors studied invariant and noninvariant hypersurfaces of almost $r$-paracontact manifolds. R. Singh [14] defined $\left(e_{1}, e_{2}, r\right)$ almost contact structure as a generalization of many known structures, which are obtained by taking particular values of $\left(e_{1}, e_{2}\right)$ and $r$ (see also [15]). The study of Lorentzian almost paracontact manifolds was initiated by K. Matsumoto in 1989 [9]. Also he introduced the notion of Lorentzian paraSasakian (for short, LP-Sasakian) manifold. I. Mihai and R. Rosca [11] defined the same notion independently and thereafter many authors ([18], [12], [16], [17]) studied Lorentzian para-Sasakian manifolds and their submanifolds.

In the present paper, we study the invariant and noninvariant hypersurfaces of $(1,1,1)$ almost contact manifolds, Lorentzian almost paracontact manifolds and Lorentzian para-Sasakian manifolds, respectively. We investigate the invariant hypersurfaces with two different conditions: when the characteristic vector field $\xi$ is everywhere tangent to the hypersurfaces and when the characteristic vector field $\xi$ is not tangent the hypersurfaces. Section 2 is devoted to preliminaries. In Section 3 we show that a noninvariant hypersurface of an $(1,1,1)$ almost contact manifold with the characteristic vector field $\xi$ nowhere tangent to the hypersurface admits an almost product structure. In Section 4 we study hypersurfaces of affinely cosymplectic and normal $(1,1,1)$ almost contact manifolds. In Section 5 it is proved that a noninvariant hypersurface of a Lorentzian almost paracontact manifold is an almost product metric manifold. We also find a necessary and sufficient condition for a noninvariant hypersurface of a Lorentzian para-Sasakian manifold to be locally product manifold. Moreover, in this section we establish a Lorentzian para-Sasakian structure for an invariant hypersurface of a Lorentzian para-Sasakian manifold with the characteristic vector field $\xi$ tangent to the hypersurface. In the last section we give some examples for invariant and noninvariant hypersurfaces of an $(1,1,1)$ almost contact manifold, a Lorentzian almost paracontact manifold and a Lorentzian para-Sasakian manifold.

\section{Preliminaries}

Let $\bar{M}$ be an $n$-dimensional differentiable manifold. If there exist a tensor field $\varphi$ of type $(1,1), r$-linearly independent vector fields $\xi_{\alpha}$ and $r 1$-forms $\eta^{\alpha}$ on $\bar{M}$ such that [15]

$$
\begin{aligned}
\varphi\left(\xi_{\alpha}\right) & =0, \\
\varphi^{2} & =e_{1} I+e_{2} \eta^{\alpha} \otimes \xi_{\alpha},
\end{aligned}
$$

where $e_{1}, e_{2}$ take values \pm 1 independently, $I$ denotes the identity map of $\Gamma(T \bar{M})$ and $\otimes$ is the tensor product, then the structure $\left(\varphi, \xi_{\alpha}, \eta^{\alpha}\right)$ is said to be an almost $\left(e_{1}, e_{2}\right)$-r-contact structure or in short $\left(e_{1}, e_{2}, r\right)$ AC-structure 
and the manifold $\bar{M}$ with the $\left(e_{1}, e_{2}, r\right)$ AC-structure is called an $\left(e_{1}, e_{2}, r\right)$ AC-manifold.

Let $\bar{M}$ be an $\left(e_{1}, e_{2}, r\right)$ AC-manifold. Then the following relations hold on $\bar{M}$ [14]:

$$
\begin{aligned}
\eta^{\alpha} \circ \varphi & =0, \\
\eta^{\alpha}\left(\xi_{\beta}\right) & =-e_{1} e_{2} \delta_{\beta}^{\alpha}, \\
\operatorname{rank}(\varphi) & =n-r .
\end{aligned}
$$

Now, consider that $\bar{M}$ is an $(1,1,1)$ AC-manifold. Then $\bar{M}$ admits a Lorentzian metric $\bar{g}$, such that

$$
\begin{aligned}
\eta(\bar{X}) & =\bar{g}(\bar{X}, \xi), \\
\bar{g}(\varphi \bar{X}, \varphi \bar{Y}) & =\bar{g}(\bar{X}, \bar{Y})+\eta(\bar{X}) \eta(\bar{Y}),
\end{aligned}
$$

for all $\bar{X}, \bar{Y} \in \Gamma(T \bar{M})$. In this case $\bar{M}$ is said to admit a Lorentzian almost paracontact structure $(\varphi, \xi, \eta, \bar{g})$. Then we get

$$
\begin{aligned}
\Phi(\bar{X}, \bar{Y}) & \equiv \bar{g}(\bar{X}, \varphi \bar{Y}) \equiv \bar{g}(\varphi \bar{X}, \bar{Y}) \equiv \Phi(\bar{Y}, \bar{X}), \\
\left(\bar{\nabla}_{\bar{X}} \Phi\right)(\bar{Y}, \bar{Z}) & =\bar{g}\left(\bar{Y},\left(\bar{\nabla}_{\bar{X}} \varphi\right) Z\right)=\left(\bar{\nabla}_{\bar{X}} \Phi\right)(\bar{Z}, \bar{Y}),
\end{aligned}
$$

where $\bar{\nabla}$ is the Levi-Civita connection with respect to $\bar{g}$. It is clear that the Lorentzian metric $\bar{g}$ makes $\xi$ a timelike unit vector field, i.e., $\bar{g}(\xi, \xi)=-1$. The manifold $\bar{M}$ equipped with a Lorentzian almost paracontact structure $(\phi, \xi, \eta, \bar{g})$ is called a Lorentzian almost paracontact manifold (for short, LAPmanifold) [9], [19].

A Lorentzian almost paracontact manifold $\bar{M}$ endowed with the structure $(\varphi, \xi, \eta, \bar{g})$ is called a Lorentzian paracontact manifold (for short, LPmanifold) [9] if

$$
\Phi(\bar{X}, \bar{Y})=\frac{1}{2}\left(\left(\bar{\nabla}_{\bar{X}} \eta\right) \bar{Y}+\left(\bar{\nabla}_{\bar{Y}} \eta\right) \bar{X}\right) .
$$

A Lorentzian almost paracontact manifold $\bar{M}$ endowed with the structure $(\varphi, \xi, \eta, \bar{g})$ is called a Lorentzian para Sasakian manifold (for short, LPSasakian) [9] if

$$
\left(\bar{\nabla}_{\bar{X}} \varphi\right) \bar{Y}=\eta(\bar{Y}) \bar{X}+\bar{g}(\bar{X}, \bar{Y}) \xi+2 \eta(\bar{X}) \eta(\bar{Y}) \xi .
$$

We note that in a LP-Sasakian manifold the 1-form $\eta$ is closed.

Let $\bar{M} \times R$ be a product manifold, where $\bar{M}$ is an $(1,1,1)$ AC-manifold. The tensor field $J^{\prime}$ of type $(1,1)$ on $\bar{M} \times R$ defined by

$$
J^{\prime}\left(\bar{X}, f \frac{d}{d t}\right)=\left(\varphi \bar{X}-f \xi, \eta(\bar{X}) \frac{d}{d t}\right),
$$


where $f$ is a $C^{\infty}$ real-valued function and $\bar{X} \in \Gamma(T \bar{M})$, satisfies $J^{\prime 2}=I$ and thus provides an almost product structure on $\bar{M} \times R$. If the induced almost product structure on $\bar{M} \times R$ is integrable then the $(1,1,1)$ AC-structure on $\bar{M}$ is said to be normal [15]. Since the vanishing of the Nijenhuis tensor $\left[J^{\prime}, J^{\prime}\right]$ is a necessary and sufficient condition for integrability, the condition of the normality in terms of the Nijenhuis tensor $[\varphi, \varphi]$ of $\varphi$ is (see [15])

$$
[\varphi, \varphi]+d \eta \otimes \xi=0
$$

where

$$
[\varphi, \varphi](\bar{X}, \bar{Y})=[\varphi \bar{X}, \varphi \bar{Y}]-\varphi[\varphi \bar{X}, \bar{Y}]-\varphi[\bar{X}, \varphi \bar{Y}]+\varphi^{2}[\bar{X}, \bar{Y}],
$$

for all $\bar{X}, \bar{Y} \in \Gamma(T \bar{M})$.

\section{Noninvariant Hypersurfaces of $(1,1,1)$ AC-Manifolds}

Let $\bar{M}$ be an $(1,1,1)$ AC-manifold. Consider an $(n-1)$-dimensional manifold $M$ imbedded in $\bar{M}$ with the immersion $i: M \rightarrow \bar{M}$ and assume that for each $p \in M$ the vector $\xi_{i(p)}$ is not tangent to the hypersurface. Then we have

$$
\varphi i_{*} X=i_{*} J X+\alpha(X) \xi
$$

where $J$ is a tensor field of type $(1,1), \alpha$ is a 1 -form on $M$ and $i_{*}$ is the differential of the immersion $i$ of $M$ into $\bar{M}$. If $\alpha \neq 0$, then the submanifold $i(M)$ is called a noninvariant hypersurface of $\bar{M}$. On the other hand, if the 1-form $\alpha$ vanishes, then $i(M)$ is called an invariant hypersurface of $\bar{M}$ (see [7]). A hypersurface may, of course, be neither invariant nor noninvariant. Throughout this section, unless specified otherwise $i(M)$ will be a noninvariant hypersurface of the $(1,1,1)$ AC-manifold $\bar{M}$.

THEOREM 3.1. If $M$ is a noninvariant hypersurface of an $(1,1,1) A C$ manifold $\bar{M}$ with $\xi$ nowhere tangent to $M$, then $M$ admits an almost product structure.

Proof. By applying $\varphi$ to (3.1) and using (2.1)-(2.4), we have

$$
i_{*} X+\eta\left(i_{*} X\right) \xi=i_{*}\left(J^{2} X\right)+\alpha(J X) \xi .
$$

Then from (3.1), we get

$$
J^{2} X=X
$$

and

$$
\alpha(J X)=\eta\left(i_{*} X\right)=i^{*}(\eta X),
$$


where $X \in \Gamma(T M)$ and $i^{*}$ is the dual map of $i_{*}$. So $J$ acts as an almost product structure on $M$. This completes the proof.

If we define a 1-form $C \alpha$ on $M$ by $C \alpha(X)=\alpha(J X)$ then from (3.3) we can write

$$
C \alpha=i^{*} \eta
$$

Thus, the hypersurface $M$ admits a 1-form $\alpha$ whose vanishing means that the tangent hyperplane of the hypersurface is invariant under $\varphi$.

Now, let $\bar{\nabla}$ be a symmetric affine connection on $\bar{M}$ and define an affine connection $\nabla$ on $M$ with respect to the affine normal $\xi$ by

$$
\bar{\nabla}_{i_{*} X} i_{*} Y=i_{*} \nabla_{X} Y+h(X, Y) \xi,
$$

where $h$ is a symmetric tensor field of type $(0,2)$ on $M$ which is called the second fundamental form of $M$ with respect to $\xi$.

Suppose that the $(1,1,1)$ AC-structure is normal. Then, the torsion field $S$ of type $(1,2)$ on $M$ which is defined by

$$
S(\bar{X}, \bar{Y})=[\varphi \bar{X}, \varphi \bar{Y}]-\varphi[\varphi \bar{X}, \bar{Y}]-\varphi[\bar{X}, \varphi \bar{Y}]+\varphi^{2}[\bar{X}, \bar{Y}]+d \eta(\bar{X}, \bar{Y}) \xi
$$

for all $\bar{X}, \bar{Y} \in \Gamma(T \bar{M})$, vanishes. By taking $\bar{Y}=\xi$ in (3.5), we get

$$
L_{\xi} \varphi=0 \quad \text { and } \quad L_{\xi} \eta=0,
$$

where $L_{\xi}$ is the Lie derivative operator with respect to $\xi$. From (3.5) the tensor field $S$ is also expressed by

$$
\begin{aligned}
& S(\bar{X}, \bar{Y})=\bar{\nabla}_{\varphi \bar{X}}(\varphi \bar{Y})-\bar{\nabla}_{\varphi \bar{Y}}(\varphi \bar{X})-\varphi\left(\bar{\nabla}_{\varphi \bar{X}} \bar{Y}-\bar{\nabla}_{\bar{Y}}(\varphi \bar{X})\right) \\
&-\varphi\left(\bar{\nabla}_{\bar{X}}(\varphi \bar{Y})-\bar{\nabla}_{\varphi \bar{Y}} \bar{X}\right)+\varphi^{2}\left(\bar{\nabla}_{\bar{X}} \bar{Y}-\bar{\nabla}_{\bar{Y}} \bar{X}\right) \\
&+\left(\bar{\nabla}_{\bar{X}} \eta(\bar{Y})-\bar{\nabla}_{\bar{Y}} \eta(\bar{X})-\eta([\bar{X}, \bar{Y}])\right) \xi
\end{aligned}
$$

or

$$
\begin{aligned}
S(\bar{X}, \bar{Y})=\left(\bar{\nabla}_{\varphi \bar{X}} \varphi\right) \bar{Y}-\left(\bar{\nabla}_{\varphi \bar{Y}} \varphi\right) \bar{X}+\varphi\left(\bar{\nabla}_{\bar{Y}} \varphi\right) \bar{X} \\
\\
-\varphi\left(\bar{\nabla}_{\bar{X}} \varphi\right) \bar{Y}+\left[\left(\bar{\nabla}_{\bar{X}} \eta\right) \bar{Y}-\left(\bar{\nabla}_{\bar{Y}} \eta\right) \bar{X}\right] \xi .
\end{aligned}
$$

By using (3.1) and (3.4), we obtain

$$
\begin{aligned}
S\left(i_{*} X, i_{*} Y\right)=i_{*}[ & J, J](X, Y)+L_{\xi} \varphi\left\{\alpha(X) i_{*} Y-\alpha(Y) i_{*} X\right\} \\
& +\left\{d \alpha(J X, Y)+d \alpha(X, J Y)-2 i^{*} \eta([X, Y])\right\} \xi .
\end{aligned}
$$

Therefore, we have the following: 
THEOREM 3.2. A noninvariant hypersurface of a normal $(1,1,1)$ AC-manifold $\bar{M}$ is a locally product manifold which has a 1 -form $\alpha=C^{-1} i^{*} \eta$ such that its differential satisfies

$$
d \alpha(J X, Y)+d \alpha(X, J Y)=2 C \alpha([X, Y]) .
$$

COROLlary 3.3. An invariant hypersurface of an $(1,1,1)$ AC-manifold is an almost product manifold. If the $(1,1,1) A C$-manifold is normal, then the almost product structure is integrable.

THEOREM 3.4. Let $\xi$ be an infinitesimal automorphism of the $(1,1,1)$ $A C$-manifold $\bar{M}$. If, for every noninvariant hypersurface, the induced almost product structure $J$ is integrable and the differential of the induced 1-form $\alpha=C^{-1} i^{*} \eta$ satisfies (3.9) then $\bar{M}$ is normal.

\section{Hypersurfaces of affinely cosymplectic and normal $(1,1,1) \mathrm{AC}$ - manifolds}

Let $\bar{M}$ be an $(1,1,1)$ AC-manifold with a symmetric affine connection $\bar{\nabla}$ and $\nabla$ denotes the induced connection on the noninvariant hypersurface $M$. If we write

$$
\left(\nabla_{X} i_{*}\right) Y=\bar{\nabla}_{i_{*} X} i_{*} Y-i_{*}\left(\nabla_{X} Y\right),
$$

then the Gauss and Weingarten equations are

$$
\left(\nabla_{X} i_{*}\right) Y=h(X, Y) \xi, \quad h(X, Y)=h(Y, X)
$$

and

$$
\bar{\nabla}_{i_{*} X} \xi=-i_{*} A X+w(X) \xi,
$$

where $h$ and $A$ are the second fundamental tensors of type $(0,2)$ and $(1,1)$, respectively of $M$ with respect to $\xi$, and $w$ is a 1 -form on $M$ defining the connection on the affine normal bundle.

By using (3.1), (4.2) and (4.3) we get

$$
\begin{aligned}
\left(\bar{\nabla}_{i_{*} X} \varphi\right) i_{*} Y= & \bar{\nabla}_{i_{*} X} \varphi i_{*} Y-\varphi\left(\bar{\nabla}_{i_{*} X} i_{*} Y\right) \\
= & {\left[h(X, J Y)+\left(\nabla_{X} \alpha\right)(Y)+w(X) \alpha(Y)\right] \xi } \\
& \quad+i_{*}\left[\left(\nabla_{X} J\right) Y-\alpha(Y) A X\right] .
\end{aligned}
$$

Then we will investigate the following two cases: 
Case I: Let $\bar{M}$ be an affinely cosymplectic $(1,1,1)$ AC-manifold, that is, $\bar{M}$ be an $(1,1,1)$ AC-manifold with a symmetric affine connection $\bar{\nabla}$ such that

$$
\bar{\nabla} \varphi=0, \quad \bar{\nabla} \eta=0 .
$$

From (3.7) we can easily see that an affinely cosymplectic $(1,1,1)$ AC-manifold is normal. Also by using (2.1) and (2.2), we can show that (4.5) implies that

$$
\bar{\nabla} \xi=0 .
$$

Therefore, by (4.3), we have

$$
A X=0 \quad \text { and } \quad w(X)=0 .
$$

Moreover, since $\bar{\nabla} \varphi=0$ then from (4.4) we have

$$
\nabla J=0 \quad \text { and } \quad\left(\nabla_{X} \alpha\right)(Y)=-h(X, J Y) .
$$

Case II: Let $\bar{M}$ be a normal $(1,1,1)$ AC-manifold such that $\varphi=\bar{\nabla} \xi$. Then by using (3.1) and (4.3), we have

$$
i_{*} J X+\alpha(X) \xi=-i_{*} A X+w(X) \xi,
$$

that is, $J=-A$ and $\alpha=w$.

If $A X=0$, for all $X \in \Gamma(T M)$, then from (4.3) it is obvious that $\bar{\nabla}_{i_{*} X} \xi$ and $\xi$ are proportional. So affine normals are parallel along the hypersurface. In this case, the hypersurface $M$ is said to be totally flat.

Proposition 4.1. Let $M$ be a noninvariant hypersurface of an affinely cosymplectic $(1,1,1)$ AC-manifold. Then $M$ is totally flat and

$$
\nabla J=0, \quad\left(\nabla_{X} \alpha\right)(Y)=-h(X, J Y), \quad w=0 .
$$

COROLLARY 4.2. Let $M$ be an invariant hypersurface of an affinely cosymplectic $(1,1,1)$ AC-manifold. Then

$$
\nabla J=0, \quad h=0, \quad w=0 .
$$

PRoposition 4.3. Let $M$ be a noninvariant hypersurface of a normal $(1,1,1)$ AC-manifold such that $\varphi=\bar{\nabla} \xi$. Then

$$
J=-A \quad \text { and } \quad \alpha=w .
$$




\section{Hypersurfaces of Lorentzian almost paracontact manifolds}

An $(1,1,1)$ AC-manifold $\bar{M}$ admitting a Lorentzian metric $\bar{g}$ such that

$$
\begin{aligned}
\bar{g}(\bar{X}, \xi) & =\eta(\bar{X}), \\
\bar{g}(\bar{X}, \varphi \bar{Y}) & \equiv \bar{g}(\varphi \bar{X}, \bar{Y}),
\end{aligned}
$$

where $\bar{X}, \bar{Y} \in \Gamma(T \bar{M})$, is called Lorentzian almost paracontact manifold and denoted by $(\bar{M}, \varphi, \eta, \bar{g})$.

Proposition 5.1. Let $(M, J, \alpha, g)$ be a noninvariant hypersurface of $(\bar{M}, \varphi, \eta, \bar{g})$ where $g$ is the induced metric on $M$, that is, $i^{*} \bar{g}=g$. Then the hypersurface $(M, J, \alpha, g)$ admits an almost product metric

$$
G=g+\alpha \otimes \alpha .
$$

Proof. From (5.2), we can write

$$
\bar{g}\left(\varphi i_{*} X, i_{*} Y\right)=\bar{g}\left(\varphi i_{*} X, i_{*} Y\right) .
$$

By using (2.1) in (5.4), we obtain

$$
\bar{g}\left(i_{*} J X, i_{*} Y\right)+\alpha(X) \eta\left(i_{*} Y\right)=\bar{g}\left(i_{*} X, i_{*} J Y\right)+\alpha(Y) \eta\left(i_{*} X\right) .
$$

The induced metric $g$ on $(M, J, \alpha)$ can be defined by

$$
g(X, Y)=\bar{g}\left(i_{*} X, i_{*} Y\right) .
$$

So if we use (3.3) and (5.4) in (5.5), then we have

$$
g(J X, Y)+\alpha(X) C \alpha(Y)=g(X, J Y)+\alpha(Y) C \alpha(X),
$$

that is,

$$
(g+\alpha \otimes \alpha)(J X, Y)=(g+\alpha \otimes \alpha)(X, J Y) .
$$

If we denote $g+\alpha \otimes \alpha$ by $G$, the proof is completed.

COROLlaRY 5.2. A noninvariant hypersurface of a Lorentzian almost paracontact manifold is an almost product metric manifold.

Now, let define 2-forms

$$
\Phi(\bar{X}, \bar{Y})=\bar{g}(\varphi \bar{X}, \bar{Y}), \quad \bar{X}, \bar{Y} \in \Gamma(T \bar{M})
$$

and

$$
\Omega(X, Y)=G(J X, Y), \quad X, Y \in \Gamma(T M) .
$$


$\Phi$ and $\Omega$ are called the fundamental forms of the Lorentzian almost paracontact manifold $(\bar{M}, \varphi, \eta, \bar{g})$ and the submanifold $(M, J, G)$ of $\bar{M}$, respectively. Then we have the following:

LEMmA 5.3. Let $\Phi$ and $\Omega$ be the fundamental forms of $(\bar{M}, \varphi, \eta, \bar{g})$ and $(M, J, \alpha, G)$, respectively. Then

$$
i^{*} \Phi=\Omega-C \alpha \wedge \alpha
$$

Proof. For $X, Y \in \Gamma(T M)$, by using definitions of the fundamental forms, (3.1) and (5.3), we get

$$
\Phi\left(i_{*} X, i_{*} Y\right)=\Omega(X, Y)-(C \alpha \wedge \alpha)(X, Y) .
$$

Hence, we obtain

$$
i^{*} \Phi(X, Y)=(\Omega-C \alpha \wedge \alpha)(X, Y) .
$$

THeOREM 5.4. Let $(M, J, \alpha, G)$ be a noninvariant hypersurface of the Lorentzian para-Sasakian manifold $(\bar{M}, \varphi, \eta, \bar{g})$. Then

(a) $J=-A$,

(b) $\alpha=w$.

Proof. Since $(\bar{M}, \varphi, \eta, \bar{g})$ is a Lorentzian para-Sasakian manifold, we have

$$
\bar{\nabla}_{i_{*} X} \xi=\varphi i_{*} X .
$$

By using (4.3) and (3.1), we get

$$
-i_{*} A X+w(X) \xi=i_{*} J X+\alpha(X) \xi,
$$

which completes the proof.

THEOREM 5.5. If $M$ is a noninvariant hypersurface of a Lorentzian paraSasakian manifold $(\bar{M}, \varphi, \eta, \bar{g})$, then

(a) $\left(\nabla_{X} J\right)(Y)=\alpha(Y) J X-C \alpha(Y) X$,

(b) $\bar{g}\left(i_{*} X, i_{*} Y\right)+2 C \alpha(X) C \alpha(Y)=h(X, J Y)+\left(\nabla_{X} \alpha\right)(Y)+\alpha(X) \alpha(Y)$.

Proof. By using (3.1) and (4.1) we obtain

$$
\begin{aligned}
\left(\bar{\nabla}_{i_{*} X} \varphi\right)\left(i_{*} Y\right)=[ & \left.i_{*}\left(\nabla_{X} J\right)(Y)+\alpha(Y) i_{*} J X\right] \\
& +\left[h(X, J Y)+\left(\nabla_{X} \alpha\right)(Y)+\alpha(X) \alpha(Y)\right] \xi .
\end{aligned}
$$


On the other hand, since $(\bar{M}, \varphi, \eta, \bar{g})$ is a Lorentzian para-Sasakian manifold, from (2.11) we also have

$$
\left(\bar{\nabla}_{i_{*} X} \varphi\right)\left(i_{*} Y\right)=\eta\left(i_{*} Y\right) i_{*} X+\bar{g}\left(i_{*} X, i_{*} Y\right) \xi+2 \eta\left(i_{*} X\right) \eta\left(i_{*} Y\right) \xi
$$

By considering $C \alpha=i^{*} \eta$ and equating the components of (5.7) and (5.8), we get (a) and (b) in the assertion theorem. This completes the proof.

As an immediate consequence we have the following:

COROLlaRY 5.6. Let $M$ be a noninvariant hypersurface of the Lorentzian para-Sasakian manifold $(\bar{M}, \varphi, \eta, \bar{g})$ with the induced almost product structure $J$. Then $M$ is a locally product manifold if and only if

$$
\alpha(Y) J X=\alpha(J Y) X \text {. }
$$

Now, let $\bar{M}$ be a $(1,1,1)$ AC-manifold and $M$ be an invariant hypersurface of $\bar{M}$. Assume that for each $p \in M$ the vector $\xi_{i(p)}$ belongs to the tangent hyperplane of the hypersurface. For an invariant hypersurface of an $(1,1,1)$ AC-manifold we can write

$$
\varphi i_{*} X=i_{*} \psi X
$$

where $\psi$ is a tensor of type $(1,1)$ on the hypersurface $M$ and $X \in \Gamma(T M)$. Applying $\varphi$ to the both sides of the equation (5.10), we get

$$
i_{*} \psi^{2} X=\varphi^{2} i_{*} X=i_{*} X+\eta\left(i_{*} X\right) \xi .
$$

If we denote

$$
i_{*} \xi^{*}=\xi
$$

and

$$
\eta^{*}(X)=\eta\left(i_{*} X\right)
$$

then we have

$$
\psi^{2} X=X+\eta^{*}(X) \xi^{*}
$$

Furthermore,

$$
\begin{aligned}
\eta^{*}(\psi X) & =\eta\left(i_{*} \psi X\right)=\eta\left(\varphi i_{*} X\right)=0, \\
\eta^{*}\left(\xi^{*}\right) & =\eta\left(i_{*} \xi^{*}\right)=\eta(\xi)=-1
\end{aligned}
$$


and

$$
i_{*} \psi \xi^{*}=\varphi i_{*} \xi^{*}=\varphi \xi=0
$$

that is,

$$
\psi \xi^{*}=0
$$

Thus we have

THEOREM 5.7. Let $M$ be an invariant hypersurface of an $(1,1,1) A C$ manifold $(\bar{M}, \varphi, \eta, \xi)$ and $\xi \in \Gamma(T M)$. Then $M$ is an $(1,1,1)$ AC-manifold with the structure $\left(\psi, \xi^{*}, \eta^{*}\right)$ where $i_{*} \xi^{*}=\xi$ and $\eta^{*}(X)=\eta\left(i_{*} X\right)$, for all $X \in \Gamma(T M)$.

THeOREM 5.8. Let $M$ be an invariant hypersurface of an $(1,1,1) A C$ manifold $(\bar{M}, \varphi, \eta, \xi)$ with $\xi \in \Gamma(T M)$. If $\bar{M}$ is normal, then $M$ is also normal.

Proof. By using (3.5), we can write

$$
\begin{aligned}
S\left(i_{*} X, i_{*} Y\right)= & {[\varphi, \varphi]\left(i_{*} X, i_{*} Y\right)+d \eta\left(i_{*} X, i_{*} Y\right) \xi } \\
= & {\left[\varphi i_{*} X, \varphi i_{*} Y\right]-\varphi\left[\varphi i_{*} X, i_{*} Y\right]-\varphi\left[i_{*} X, \varphi i_{*} Y\right] } \\
& +\varphi^{2}\left[i_{*} X, i_{*} Y\right]+d \eta\left(i_{*} X, i_{*} Y\right) \xi .
\end{aligned}
$$

for all $X, Y \in \Gamma(T M)$. If we use (5.10), (5.12) and (5.13) in (5.18), we get

$$
\begin{aligned}
S\left(i_{*} X, i_{*} Y\right)= & i_{*} \psi^{2}[X, Y]+\left[i_{*} \psi X, i_{*} \psi Y\right]-i_{*} \psi[X, \psi Y]-i_{*} \psi[\psi X, Y] \\
& +\left\{\left(i_{*} X\right)\left(\eta^{*}(Y)\right)-\left(i_{*} Y\right)\left(\eta^{*}(X)\right)-\eta^{*}([X, Y])\right\} i_{*} \xi^{*} \\
= & i_{*}\left\{[\psi, \psi](X, Y)+d \eta^{*}(X, Y) \xi^{*}\right\} .
\end{aligned}
$$

Hence, we have the assertion of the theorem.

THEOREM 5.9. Let $M$ be an invariant hypersurface of a Lorentzian almost paracontact manifold $(\bar{M}, \varphi, \eta, \bar{g})$ where $\xi \in \Gamma(T M)$. Then $M$ is also a Lorentzian almost paracontact manifold.

Proof. From Theorem 5.7 it follows that an invariant hypersurface $M$ of $\bar{M}$ is an $(1,1,1)$ AC-manifold with the structure $\left(\psi, \xi^{*}, \eta^{*}\right)$. Let $g^{*}$ be the induced metric on $M$. Then we have

$$
g^{*}(\psi X, \psi Y)=\bar{g}\left(i_{*} \psi X, i_{*} \psi Y\right)=\bar{g}\left(\varphi i_{*} X, \varphi i_{*} Y\right) .
$$

Since $\bar{M}$ is a Lorentzian almost paracontact manifold, then by using (5.13) in (5.19) we get

$$
g^{*}(\psi X, \psi Y)=g^{*}(X, Y)+\eta^{*}(X) \eta^{*}(Y) .
$$


Moreover,

$$
g^{*}\left(X, \xi^{*}\right)=\bar{g}\left(i_{*} X, i_{*} \xi^{*}\right)=\eta\left(i_{*} X\right)=\eta^{*}(X),
$$

which completes the proof.

THEOREM 5.10. Let $(\bar{M}, \varphi, \eta, \bar{g})$ be a Lorentzian para Sasakian manifold. Then an invariant hypersurface with $\xi \in \Gamma(T M)$ of $\bar{M}$ is also a Lorentzian para Sasakian manifold.

Proof. Let $\bar{M}$ be a Lorentzian para-Sasakian manifold. Then we have

$$
\bar{\nabla}_{i_{*} X} \xi=\varphi i_{*} X
$$

where $\bar{\nabla}$ is a Levi-Civita connection with respect to $\bar{g}$. From (5.10) and (5.12), we can write

$$
\bar{\nabla}_{i_{*} X} i_{*} \xi^{*}=i_{*} \psi X
$$

By using (3.4) in the last equation, we obtain

$$
i_{*} \nabla_{X} \xi^{*}+h\left(X, \xi^{*}\right) N=i_{*} \psi X
$$

where $\nabla$ is the induced connection on $M$ and $N$ is normal to $M$. If we consider normal and tangent components of above equation we get

$$
\begin{aligned}
\nabla_{X} \xi^{*} & =\psi X, \\
h\left(X, \xi^{*}\right) & =0 .
\end{aligned}
$$

Since $\bar{M}$ be a Lorentzian para Sasakian manifold from (2.11), we have

$$
\left(\bar{\nabla}_{i_{*} X} \varphi\right) i_{*} Y=\eta\left(i_{*} Y\right) i_{*} X+\bar{g}\left(i_{*} X, i_{*} Y\right) \xi+2 \eta\left(i_{*} X\right) \eta\left(i_{*} Y\right) \xi,
$$

for all $X, Y \in \Gamma(T M)$. By using (5.10), (5.12) and (5.13) in (5.22), we obtain

$$
\left(\bar{\nabla}_{i_{*} X} \varphi\right) i_{*} Y=i_{*}\left\{\eta^{*}(Y) X+\bar{g}(X, Y) \xi^{*}+2 \eta^{*}(X) \eta^{*}(Y) \xi^{*}\right\} .
$$

On the other hand, from (3.4) and (5.10), one can get

$$
\begin{aligned}
\left(\bar{\nabla}_{i_{*} X} \varphi\right) i_{*} Y & =\bar{\nabla}_{i_{*} X} \varphi i_{*} Y-\varphi\left(\bar{\nabla}_{i_{*} X} i_{*} Y\right) \\
& =\bar{\nabla}_{i_{*} X} i_{*} \psi Y-\varphi\left(i_{*} \nabla_{X} Y+h(X, Y) N\right) \\
& =i_{*}\left(\nabla_{X} \psi Y-\psi\left(\nabla_{X} Y\right)\right)+h(X, \psi Y) N-h(X, Y) \varphi N,
\end{aligned}
$$

where $\nabla$ is the induced connection on $M$ and $N$ is normal to $M$. By equating right hand sides of equations (5.23) and (5.24), we have

$$
\left(\nabla_{X} \psi\right) Y=\eta^{*}(Y) X+\bar{g}(X, Y) \xi^{*}+2 \eta^{*}(X) \eta^{*}(Y) \xi^{*} .
$$


This completes the proof.

\section{Examples}

Example 6.1. Let $\bar{M}$, be the 5-dimensional real number space with a coordinate system $(x, y, z, t, s)$. Defining

$$
\begin{gathered}
\eta=d s-d x-d z, \quad \xi=-\frac{\partial}{\partial s}, \\
\varphi\left(\frac{\partial}{\partial x}\right)=-\frac{\partial}{\partial x}-\frac{\partial}{\partial s}, \quad \varphi\left(\frac{\partial}{\partial y}\right)=-\frac{\partial}{\partial y} \\
\varphi\left(\frac{\partial}{\partial z}\right)=-\frac{\partial}{\partial z}-\frac{\partial}{\partial s}, \quad \varphi\left(\frac{\partial}{\partial t}\right)=-\frac{\partial}{\partial t}, \quad \varphi\left(\frac{\partial}{\partial s}\right)=0,
\end{gathered}
$$

the set $(\varphi, \xi, \eta)$ becomes a $(1,1,1)$ AC-structure in $\bar{M}$.

Let $M_{1}$ be a hypersurface of $\bar{M}$ which is given by $s=x$ with the immersion $i: M_{1} \rightarrow \bar{M}$. Then

$$
\begin{aligned}
\left\{u_{1}=(1,0,0,0,1), u_{2}=\right. & (0,1,0,0,0), \\
& \left.u_{3}=(0,0,1,0,0), u_{4}=(0,0,0,1,0)\right\}
\end{aligned}
$$

is a local basis for the tangent hyperplane of $M_{1}$ and $N_{1}=(1,0,0,0,-1)$ is the normal vector field of the hypersurface. It is obvious that the characteristic vector field $\xi_{i(p)}, p \in M_{1}$, is not tangent to hypersurface of $M_{1}$. A tangent vector field of the hypersurface can be written by $X \equiv i_{*} X=f_{1} u_{1}+f_{2} u_{2}+$ $f_{3} u_{3}+f_{4} u_{4}$ for some smooth functions $f_{i}, 1 \leq i \leq 4$, on $M$. Then we have

$$
\varphi i_{*} X=-f_{1} u_{1}-f_{2} u_{2}-f_{3} u_{3}-f_{4} u_{4}+f_{3} \xi,
$$

which shows that $M_{1}$ is a noninvariant hypersurface of $\bar{M}$.

Now let us consider the hypersurface $M_{2}$ of the $(1,1,1)$ AC-manifold $\bar{M}$ defining by $x=y$ and let $i: M_{2} \rightarrow \bar{M}$ be the immersion of $M_{2}$ into $M$. In this case the set

$$
\begin{aligned}
\left\{v_{1}=(1,1,0,0,0), v_{2}=\right. & (0,0,1,0,0), \\
& \left.v_{3}=(0,0,0,1,0), v_{4}=(0,0,0,0,1)\right\}
\end{aligned}
$$

is a local basis for the tangent hyperplane and $N_{2}=(1,-1,0,0,0)$ is the normal vector field of $M_{2}$. The characteristic vector field is tangent to the the hypersurface. For any tangent vector field $X \equiv i_{*} X=h_{1} v_{1}+h_{2} v_{2}+h_{3} v_{3}+$ $h_{4} v_{4}$ of the hypersurface we have

$$
\varphi i_{*} X=-h_{1} v_{1}-h_{2} v_{2}-f_{3} v_{3}+\left(h_{1}+h_{2}\right) \xi,
$$


where $h_{i}, 1 \leq i \leq 4$, are some smooth functions on $M_{2}$. From (6.1) we see that $M_{2}$ is an invariant hypersurface of $\bar{M}$.

Example 6.2. Let $\bar{M}$ be the 5-dimensional real number space with a coordinate system $(x, y, z, t, s)$. In $\bar{M}$ we define

$$
\begin{gathered}
\eta=d s-d x, \quad \xi=-\frac{\partial}{\partial s}, \\
\varphi\left(\frac{\partial}{\partial x}\right)=\frac{\partial}{\partial x}+\frac{\partial}{\partial s}, \quad \varphi\left(\frac{\partial}{\partial y}\right)=\frac{\partial}{\partial y}, \\
\varphi\left(\frac{\partial}{\partial z}\right)=\frac{\partial}{\partial z}, \quad \varphi\left(\frac{\partial}{\partial t}\right)=\frac{\partial}{\partial t}, \quad \varphi\left(\frac{\partial}{\partial s}\right)=0, \\
g=(d x)^{2}+(d y)^{2}+(d z)^{2}+(d t)^{2}-\eta \otimes \eta .
\end{gathered}
$$

Then $(\varphi, \xi, \eta, g)$ is a Lorentzian almost paracontact structure in $\bar{M}$.

Let $M$ be a hypersurface of $\bar{M}$ which is defined by $s=x$ with the immersion $i: M \rightarrow \bar{M}$. Then the set

$$
\begin{aligned}
\left\{u_{1}=(1,0,0,0,1), u_{2}=\right. & (0,1,0,0,0), \\
& \left.u_{3}=(0,0,1,0,0), u_{4}=(0,0,0,1,0)\right\}
\end{aligned}
$$

is a local basis for the tangent hyperplane of $M$ and $N=(1,0,0,0,-1)$ is the normal vector field of the hypersurface. Since $\xi_{i(p)}=\frac{1}{2}\left(u_{1}-N\right)_{i(p)}$, it can be easily seen that the characteristic vector field $\xi_{i(p)}, p \in M$, is not tangent to $M$. Moreover, since $\varphi u_{1}=u_{1}, \varphi u_{2}=u_{2}, \varphi u_{3}=u_{3}, \varphi u_{4}=u_{4}$, then $M$ is an invariant hypersurface of $\bar{M}$ with the characteristic vector field $\xi_{i(p)}, p \in M$, which is not tangent to the hypersurface.

Example 6.3. Let $\bar{M}$ be the 3-dimensional real number space with a coordinate system $(x, y, z)$. If we define

$$
\begin{gathered}
\eta=d z, \quad \xi=-\frac{\partial}{\partial z}, \\
\varphi\left(\frac{\partial}{\partial x}\right)=-\frac{\partial}{\partial x}, \quad \varphi\left(\frac{\partial}{\partial y}\right)=-\frac{\partial}{\partial y}, \quad \varphi\left(\frac{\partial}{\partial s}\right)=0, \\
g=(d x)^{2}+(d y)^{2}-\eta \otimes \eta .
\end{gathered}
$$

on $\bar{M}$, then $(\varphi, \xi, \eta, g)$ is a Lorentzian almost paracontact structure in $\bar{M}$.

Assume that $M$ be a surface of $\bar{M}$ given by $x=\arcsin y$ with the immersion $i: M \rightarrow \bar{M}$. Then

$$
\left\{u_{1}=\left(1, \sqrt{1-y^{2}}, 0\right), u_{2}=(0,0,1)\right\}
$$


forms a local basis for the tangent plane of $M$ and $N=\left(\sqrt{1-y^{2}},-1,0\right)$ is the normal vector field of the surface. For any tangent vector field $X$ of the surface we have

$$
\varphi i_{*} X=-f_{1} u_{1},
$$

where $X \equiv i_{*} X=f_{1} u_{1}+f_{2} u_{2}$ for some smooth functions $f_{1}, f_{2}$ on $M$. From (6.2) we obtain that $M$ is an invariant surface of $\bar{M}$ with the characteristic vector field $\xi_{i(p)}, p \in M$, belonging to the tangent plane of the surface.

EXAMPLE 6.4. Let $\bar{M}=R^{3}$ be the 3-dimensional real number space with a coordinate system $(x, y, z)$. We define

$$
\begin{gathered}
\eta=d z, \quad \xi=-\frac{\partial}{\partial z} \\
\varphi\left(\frac{\partial}{\partial x}\right)=\frac{\partial}{\partial x}, \quad \varphi\left(\frac{\partial}{\partial y}\right)=-\frac{\partial}{\partial y}, \quad \varphi\left(\frac{\partial}{\partial z}\right)=0, \\
g=e^{-2 z}(d x)^{2}+e^{2 z}(d y)^{2}-(d z)^{2} .
\end{gathered}
$$

Then $(\varphi, \xi, \eta, g)$ is a Lorentzian para-Sasakian structure on $\bar{M}$.

Let $M_{1}$ be a surface of $\bar{M}$ with the immersion $i: M_{1} \rightarrow \bar{M}$ which is given by

$$
z=x+y .
$$

Then $u_{1}=(1,0,1), u_{2}=(0,1,1)$ is a local basis for the tangent plane of the surface. The vector field

$$
N=\left(e^{2(x+y)}, e^{2(x+y)}, 1\right)
$$

is a normal vector field of $M_{1}$. Since

$$
\xi=-\frac{1}{e^{2(x+y)}+e^{-2(x+y)}-1}\left(\left(e^{2(x+y)}\right) u_{1}+\left(e^{-2(x+y)}\right) u_{2}-N\right)
$$

then for each $p \in M_{1}$ the characteristic vector field $\xi_{i(p)}$ is not tangent to the surface. A tangent vector field of the surface can be written by $X \equiv i_{*} X=$ $f_{1} u_{1}+f_{2} u_{2}$ for some smooth functions $f_{1}, f_{2}$ on $M$. By using (6.3) we have

$$
\varphi i_{*} X=f_{1} u_{1}-f_{2} u_{2}+\left(f_{1}-f_{2}\right) \xi .
$$

From (3.1) and (6.4) we get

$$
i_{*} J X=f_{1} u_{1}-f_{2} u_{2}
$$


and

$$
\alpha(X)=f_{1}-f_{2},
$$

where $J$ acts an almost product structure on $M_{1}$. Consequently, $M_{1}$ is a noninvariant surface of the Lorentzian para-Sasakian manifold $\bar{M}$ with $\xi$ nowhere tangent to $M_{1}$.

Let $M_{2}$ be another surface of $\bar{M}$ which is given by

$$
x=\arctan y .
$$

Then $v_{1}=\left(\frac{1}{1+y^{2}}, 1,0\right), v_{2}=(0,0,1)$ forms a local orthogonal basis for the tangent plane of the surface and

$$
N=\left(e^{2 z},-\frac{1}{1+y^{2}} e^{-2 z}, 0\right)
$$

is a normal vector field of $M_{2}$. It is obvious that the characteristic vector field of the manifold is tangent to the surface $M_{2}$. For any tangent vector field $i_{*} Y \equiv Y$ of the surface where $i: M_{2} \rightarrow \bar{M}$ is an immersion into the Lorentzian paraSasakian manifold $\bar{M}$ we can write $i_{*} Y=\gamma_{1} v_{1}+\gamma_{2} v_{2}$ for some smooth functions $\gamma_{1}, \gamma_{2}$ on $M_{2}$. By using (6.3) we have

$$
\varphi i_{*} Y=-\gamma_{1}\left(v_{1}-\frac{2\left(1+y^{2}\right)}{\left(1+y^{2}\right)^{2} e^{2 z}-e^{-2 z}} N\right),
$$

which shows that $M_{2}$ is a noninvariant surface of the Lorentzian para-Sasakian manifold $\bar{M}$ with $\xi$ tangent to the surface.

\section{REFERENCES}

1. Adati, T., Hypersurfaces of almost paracontact Riemannian manifolds, TRU Math. 17 (1981), 189-198.

2. Blair, D. E., Ludden, G. D., Hypersurfaces in almost contact manifolds, Tôhuku Math. J. (2) 21 (1969), 354-362.

3. Bucki, A., Hypersurfaces of almost r-paracontact Riemannian manifolds, Tensor (N.S.) 48 (1989), 245-251.

4. Bucki, A., Miernowski, A., Invariant hypersurfaces of an almost r-paracontact manifold, Demonstratio Math. 19 (1986), 113-121.

5. Eum, S. S., On complex hypersurfaces in normal almost contact spaces, Tensor (N.S.) 19 (1968), 45-50.

6. Gill, H., Dube, K. K., Invariant and Non Invariant Hypersurfaces of Almost R-Paracontact Manifolds, pp. 411-420 in: Proceedings of the National Conference on Frontiers in Applied and Computational Mathematics (FACM-2005), Allied Publishers, New Delhi 2005.

7. Goldberg, S. I., Yano, K., Noninvariant hypersurfaces of almost contact manifolds, J. Math. Soc. Japan 22 (1970), 25-34. 
8. Ludden, G. D., Submanifolds of cosymplectic manifolds, J. Differential Geometry 4 (1970), 237-244.

9. Matsumoto, K., On Lorentzian paracontact manifolds, Bull. Yamagata Univ. Natur. Sci. 12 (1989), 151-156.

10. Mihai, I., Matsumoto, K., Submanifolds of an almost $r$-paracontact Riemannian manifold of P-Sasakian type, Tensor (N.S.) 48 (1989), 136-142.

11. Mihai, I., Rosca, R., On Lorentzian P-Sasakian manifolds, pp. 155-169 in: Classical Analysis, Proc. Kazimierz Dolny 1991, World Scientific, Singapore 1992.

12. Matsumoto, K., Shaikh, A. A., and De, U. C., On Lorentzian para-Sasakian manifolds, Rend. Semin. Mat. Messina (II) (2000), suppl. 149-158.

13. Satō, I., On a structure similar to the almost contact structure, Tensor (N.S.) 30 (1976), 219-224.

14. Singh, R., Almost $\left(e_{1}, e_{2}\right)$-r-contact manifolds and their product with the Euclidean space $E^{r}$, Chapter 8, Ph.D. Thesis, Lucknow University, India 1982.

15. Singh, K. D., Tripathi, M. M., On normal ( $\left.e_{1}, e_{2}, r\right)$-ac structure, Ganita 40 (1989), 101-112.

16. Tripathi, M. M., De, U. C., Lorentzian almost paracontact manifolds and their submanifolds, J. Korea Soc. Math. Educ. 2 (2001), 101-125.

17. Tripathi, M. M., Shukla, S. S., On submanifolds of Lorentzian almost paracontact manifolds, Publ. Math. Debrecen 59 (2001), 327-338.

18. Matsumoto, K., Mihai, I., On a certain transformation in a Lorentzian para-Sasakian manifold, Tensor (N.S.) 47 (1988), 189-197.

19. Matsumoto, K., Mihai, I., Rosca, R., $\xi$-null geodesic gradient vector fields on a Lorentzian para-Sasakian manifold, J. Korean Math. Soc. 32 (1995), 17-31.

\author{
INÖNÜ UNIVERSITY \\ DEPARTMENT OF MATHEMATICS \\ FACULTY OF ARTS AND SCIENCES \\ 44280, MALATYA \\ TURKEY \\ E-mail: selcenyuksel@inonu.edu.tr \\ ekilic@inonu.edu.tr \\ skeles@inonu.edu.tr
}

